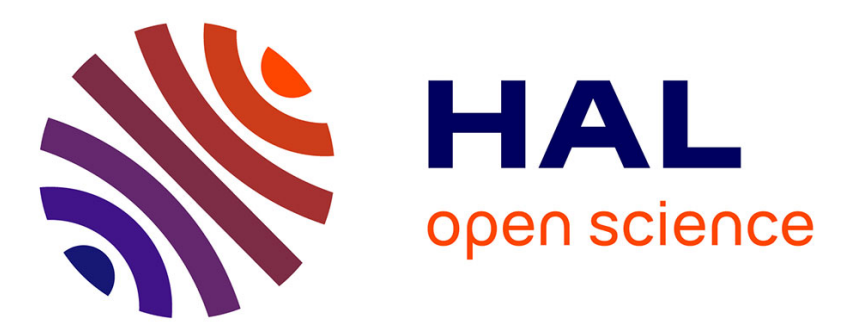

\title{
Leaderless consensus formation control of cooperative multi-agent vehicles without velocity measurements
}

\author{
Emmanuel Nuño, Antonio Loria, Elena Panteley
}

\section{To cite this version:}

Emmanuel Nuño, Antonio Loria, Elena Panteley. Leaderless consensus formation control of cooperative multi-agent vehicles without velocity measurements. IEEE Control Systems Letters, 2022, 6, pp.902-907. 10.1109/LCSYS.2021.3087095 . hal-03442759

\section{HAL Id: hal-03442759 \\ https://hal.science/hal-03442759}

Submitted on 23 Nov 2021

HAL is a multi-disciplinary open access archive for the deposit and dissemination of scientific research documents, whether they are published or not. The documents may come from teaching and research institutions in France or abroad, or from public or private research centers.
L'archive ouverte pluridisciplinaire $\mathbf{H A L}$, est destinée au dépôt et à la diffusion de documents scientifiques de niveau recherche, publiés ou non, émanant des établissements d'enseignement et de recherche français ou étrangers, des laboratoires publics ou privés. 


\title{
Leaderless consensus formation control of cooperative multi-agent vehicles without velocity measurements
}

\author{
Emmanuel Nuño Antonio Loría Elena Panteley
}

\begin{abstract}
We address the full-consensus problem for multiagent nonholonomic systems via output feedback. That is, consensus both in position and orientation considering the latter as the measured output. The controller is dynamic, but it does not rely on a velocity estimator, it relies on a dynamic extension that has a clear physical interpretation, as a mechanical system itself. Roughly speaking, it is showed that the consensus problem may be solved indirectly, by achieving consensus of the controllers themselves and, then, coupling each of these to each vehicle, via a virtual spring. Simulation tests are provided in the present manuscript to show the performance of our proposal.
\end{abstract}

Index Terms-Formation control, persistency of excitation, output feedback, nonholonomic systems, autonomous vehicles

\section{INTRODUCTION}

$\mathbf{T}$ HERE is a broad variety of problems that pertain to cooperative multi-agent systems in robotics, particularly, in mobile robotics, such as reconnaissance missions. In that scenario, it is typically required from the vehicles to advance in formation, perhaps following a leader, virtual or otherwise. This leads to the problem of formation-tracking control for multiagent systems, which has been studied under various conditions, see for instance [1]- [6]. This problem is sometimes called leader-follower consensus - $c f$. [7], [8] as opposed to the problem of leaderless consensus, which is reminiscent of the classical consensus paradigm in which the states of a group of systems converge to a common equilibrium.

If it is expected that both the vehicles' positions and orientations converge to a common value we speak of full consensus [9], [10]; otherwise, if consensus is to be achieved only in position [11], [12] or only in orientation [6], we speak of partial consensus. Because the robots can obviously not occupy the same physical space simultaneously, a formation pattern with an unknown center must be imposed. This is done by simply specifying for each robot, an offset position from the unknown center. See Fig. 1 for an illustration.

Emmanuel Nuño is with the Department of Computer Science at the University of Guadalajara. Guadalajara, Mexico (e-mail: emmanuel.nuno@cucei.udg.mx). Antonio Loría and Elena Panteley are with Laboratoire des signaux et systèmes, CNRS, Gif-sur-Yvette, France; (e-mails: aloria@ieee.org; elena.panteley@centralesupelec.fr). Elena Panteley is also with ITMO University, St. Petersbourg, Russia.

This article is supported by the Mexican CONACyT Basic Scientific Research grant CB-282807, by the French ANR via project HANDY, number ANR-18-CE40-0010, and by CEFIPRA under the grant number 6001-A.
The significance of each of these distinct problems, leaderless and leader-follower consensus, cannot be overestimated. They are, together, constituting parts of a more complex cooperative maneuvering task [13]. For instance, leaderless consensus may be considered as a preliminary stage to formationtracking control.

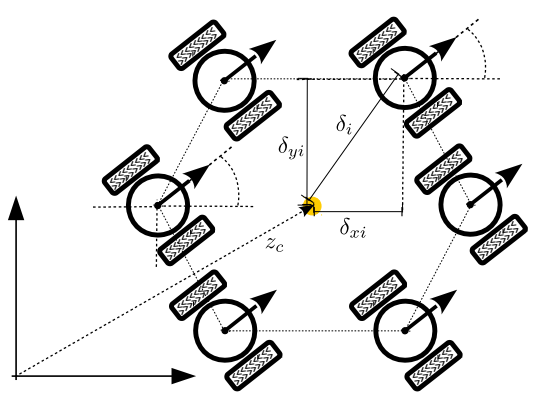

Fig. 1. Robots reaching full consensus-formation: they meet around a set-point with common orientation

In this paper we address the problem of leaderless consensus formation control for multi-vehicle systems under the following scenario. A group of mobile robots, each equipped only with position and orientation measurement sensors, is required to meet at a rendezvous point not given a priori, but which depends on the initial positions and orientations of the robots and the network topology. The robots are assumed to be able to communicate through a wireless network; not in an all-to-all topology, but each robot is connected to at least one neighbor, bidirectionally.

Our main contribution is a novel controller of remarkable simplicity. Being a dynamic system itself, the controller is reminiscent of a trivial second-order mass-spring dynamical system. The stabilization mechanism relies on the appropriate virtual coupling of each controller to a vehicle. That is, it is consensus of the controllers themselves that is achieved in the first place and, due to the virtual coupling with the controller, consensus of the vehicles follows. Consequently, our control approach obviates the use of observers.

From a systems viewpoint, the controller is dynamic and uses output feedback (the output of each robot corresponding to its position and orientation) while the problem addressed is that of consensus in the measured outputs. Such problems have been studied in the literature, e.g., for high-order linear systems [14], vehicles considered as double-integrators [1], 
[3], [15] or systems with holonomic constraints [5]. None of such appoaches, however, applies to nonholonomic systems. On the other hand, in spite of the many articles on control of nonholonomic systems, very few address the output-feedback control problem in a multiagent setting; see, e.g., [2], [16] on the leader-follower problem or [17] on leaderless consensus via output feedback, using approximate differentiation.

In the next section we present the problem formulation. In Section III we present our main result; a stability proof is provided in Section IV. Numerical illustrative simulations are presented in Section V, before concluding with some remarks in Section VI.

\section{MOdEL AND PROBLEM FORMULATION}

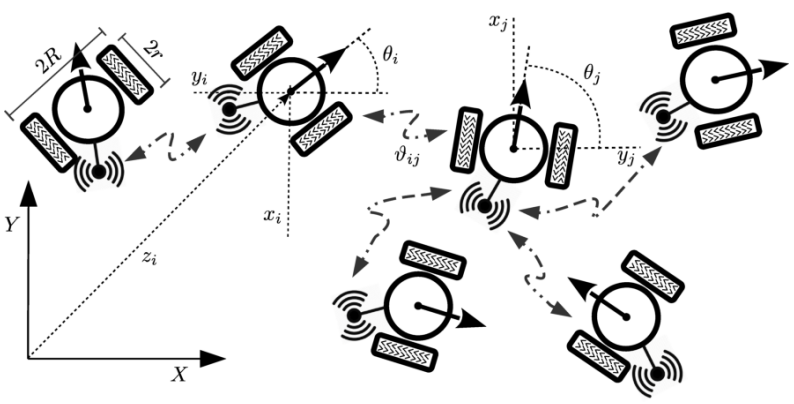

Fig. 2. Schematics of a group of differential wheeled mobile robots communicating over a wireless network.

Consider a group of $N$ force-controlled differential-drive autonomous vehicles, as depicted in Figure 2. Each of these vehicles is assumed to be torque-controlled at each wheel independently; we denote these torques $\tau_{i 1}$ and $\tau_{i 2}$. The position of the $i$ th robot on a fixed frame is given by the 2-dimensional vector of Cartesian coordinates $z_{i}:=\left[\begin{array}{ll}x_{i} & y_{i}\end{array}\right]$ and its orientation with respect to the abscissae is denoted $\theta_{i}$. Furthermore, denoting by $v_{i}$ and $\omega_{i}$ the vehicle's forward and angular velocities, respectively, the full dynamic model for the $i$ th robot is given by the following equations:

$$
\begin{gathered}
\dot{z}_{i}=\varphi_{i}\left(\theta_{i}\right) v_{i}, \quad \varphi_{i}\left(\theta_{i}\right):=\left[\cos \left(\theta_{i}\right) \sin \left(\theta_{i}\right)\right]^{\top} \\
\dot{v}_{i}=\frac{1}{r_{i} m_{i}}\left[\tau_{i 1}+\tau_{i 2}\right]=: u_{v i} \\
\dot{\theta}_{i}=\omega_{i} \\
\dot{\omega}_{i}=\frac{2 R_{i}}{I_{i} r_{i}}\left[\tau_{i 1}-\tau_{i 2}\right]=: u_{\omega i}
\end{gathered}
$$

For control design purposes, we also introduce the inputs $u_{v i}$ and $u_{\omega i}$. These quantities depend on the robot's mass $m_{i}$, on the moment of inertia, $I_{i}$, on the radius of the wheels, $r_{i}$, and on the distance between a point $Q_{i}$ at the center of the robot and the wheels, $R_{i}$. For simplicity, all robots are assumed to be identical, but this is not conservative since, as we describe below, the control approach is distributed, so each controller depends only on the parameters of the actual robot. From a dynamical systems viewpoint the model (1)-(2) consists in two second-order dynamical systems, one which represents the forward motion -Eq. (1) — and one corresponding to the orientation dynamics —Eq. (2)—. This model captures well the physics of the vehicle [18].

Then, the full leaderless consensus (or rendezvous) problem consists in requiring that all the robots acquire a desired formation pattern relatively to a static point, $z_{c}$ that is not given a priori and a common angle, $\theta_{c}$. See Fig. 1 for an illustration.

It is assumed that the network contains at least one spanning tree (any node is reachable from any other node), which is the minimal configuration to achieve consensus. Therefore, each robot communicates only with nearby neighbours - see Figure 2. It is also assumed that the communication channels are bidirectional and static. Such topology may be represented by an undirected, static and connected graph that may be modeled a constant Laplacian matrix, $L:=\left[\ell_{i j}\right] \in \mathbb{R}^{N \times N}$, where

$$
\ell_{i j}=\left\{\begin{array}{cc}
\sum_{k \in \mathcal{N}_{i}} a_{i k} & i=j \\
-a_{i j} & i \neq j,
\end{array}\right.
$$

$\mathcal{N}_{i} \subset \mathbb{Z}$ is the set of indexes corresponding to robots transmitting information to the $i$ th robot, $a_{i k}>0$ if $k \in \mathcal{N}_{i}$ and $a_{i k}=0$ otherwise. By construction, $L \mathbf{1}_{N}=0$, where $\mathbf{1}_{N}=[1 \cdots 1]^{\top}$ and $L$ is symmetric, it has a unique zeroeigenvalue, and the rest of its spectrum is strictly positive. Thus, $\operatorname{rank}(L)=N-1$.

The physical formation that the robots are required to gather into may be defined by setting distance offsets for each robot. We denote these offsets by $\delta_{x i}$ and $\delta_{y i}$. Then, for each robot we define the translated position vector $\bar{z}_{i}:=z_{i}-\delta_{i}$. Then, the consensus problem is tantamount to designing $u_{v i}$ and $u_{\omega_{i}}$ so that

$$
\begin{array}{ll}
\lim _{t \rightarrow \infty} v_{i}(t)=0, & \lim _{t \rightarrow \infty} \bar{z}_{i}(t)=z_{c}, \\
\lim _{t \rightarrow \infty} \omega_{i}(t)=0, & \lim _{t \rightarrow \infty} \theta_{i}(t)=\theta_{c} \quad \forall i \leq N,
\end{array}
$$

where $\left(z_{c}, \theta_{c}\right)$ is a unique equilibrium point for (1)-(2).

Furthermore, it is assumed that each robot is equipped only with position sensors that is, for each robot only the Cartesian coordinates $z_{i}$ and the orientation angles $\theta_{i}$ are measured and constitute the output of the system.

\section{CONTROL APPROACH}

The control design primarily relies on structural properties of the system that may be well explained via linear 2nd-order mechanical systems. Thus, to motivate and better present our control design approach and as an academic example used as a starting point for our controller design, let us consider the consensus problem for a group of $N$ simple doubleintegrator systems, each communicating with nearby agents whose indexes belong to the set $\mathcal{N}_{i}$, that is,

$$
\ddot{q}_{i}=u_{i}, \quad q_{i} \in \mathbb{R} .
$$

In these systems $q_{i}$ stands for the position and hence $\ddot{q}_{i}$ is the acceleration. For system (6) the consensus problem, which consists in making $q_{i} \rightarrow q_{c}$ and $\dot{q}_{i} \rightarrow 0$ for all $i \leq N$ for a 
non-a-priori-given set-point $q_{c}$, may be solved using the simple consensus algorithm [19]

$$
u_{i}=-d_{i} \dot{q}_{i}-p_{i} \sum_{j \in \mathcal{N}_{i}} a_{i j}\left(q_{i}-q_{j}\right) ; \quad d_{i}, p_{i}>0 .
$$

The term $p_{i} \sum_{j \in \mathcal{N}_{i}} a_{i j}\left(q_{i}-q_{j}\right)$ hinges the respective positions $q_{i}$ to each of the agent's neighbours', $q_{j}$ with $j \in \mathcal{N}_{j}$. The term $-d_{i} \dot{q}_{i}$ introduces necessary damping to ensure the asymptotic stabilization of the set $\left\{q_{i}=q_{j}=q_{c}\right\}$. This simple controller, however, relies on the assumption that $\dot{q}_{i}$ is measurable. If this is not the case, one may rely on a state estimator or on a dynamic controller to inject damping [20]. A simple choice is a controller with dynamics similar to that of the plant itself

$$
\ddot{\vartheta}_{i}+d_{i} \dot{\vartheta}_{i}+p_{i} \sum_{j \in \mathcal{N}_{i}} a_{i j}\left(\vartheta_{i}-\vartheta_{j}\right)=0 .
$$

Indeed, note that this system corresponds exactly (with obvious changes in the notation) to the closed-loop dynamics of (6) with (7). That is, Eq. (8) is reminiscent of a group of second order integrators coupled via a consensus control law hence, it is expected that $\vartheta_{i} \rightarrow \vartheta_{j} \rightarrow \vartheta_{c} \equiv$ const for all $i$, $j \leq N$. Thus, it may be reasonably conjectured that if the systems (6) are coupled with the systems (8), say via a virtual spring, consensus among the two groups of systems should be achieved. More precisely, we claim that for the systems

$$
\begin{aligned}
& \ddot{q}_{i}=u_{i} \\
& \ddot{\vartheta}_{i}+d_{i} \dot{\vartheta}_{i}+p_{i} \sum_{j \in \mathcal{N}_{i}} a_{i j}\left(\vartheta_{i}-\vartheta_{j}\right)=-u_{i}
\end{aligned}
$$

it may be achieved that $q_{i} \rightarrow q_{j} \rightarrow q_{c} \equiv$ const and $\vartheta_{i} \rightarrow$ $\vartheta_{j} \rightarrow \vartheta_{c} \equiv$ const for all $i, j \leq N$ by simply defining the coupling force $u_{i}$ as

$$
u_{i}:=-k_{v i}\left(q_{i}-\vartheta_{i}\right), \quad k_{v i}>0 .
$$

We do not prove this conjecture here, but stress it only for the purpose of motivation. An interesting related remark, however, is that the closed-loop equations resulting from using (10) in (9), correspond (again, with an obvious change of notation) to

$$
\begin{aligned}
& M_{i}\left(q_{1 i}\right) \ddot{q}_{1 i}+C_{i}\left(q_{1 i}, \dot{q}_{1 i}\right) \dot{q}_{1 i}+g_{i}\left(q_{1 i}\right)=K\left(q_{2 i}-q_{1 i}\right) \\
& \ddot{q}_{2 i}+K\left(q_{2 i}-q_{1 i}\right)=d_{i} \dot{q}_{2 i}+p_{i} \sum_{j \in \mathcal{N}_{i}} a_{i j}\left(q_{2 i}-q_{2 j}\right)
\end{aligned}
$$

with $M_{i}=I$ and $C_{i}=g_{i} \equiv 0$. The relevance of this observation is that the equations (11) correspond to those of robot manipulators with flexible joints, coupled through a standard consensus algorithm. The fact that consensus is reached for such systems stems from the main results in [21].

The control design for the second order mechanical systems, albeit with nonholonomic constraints, (1)-(2) relies on the previous observations and a cascades argument [22]. First, we design dynamic consensus algorithms separately for the forward-velocity dynamics, (1), and for the orientation dynamics, (2). The control design for each of these follows the rationale above while incorporating the additional difficulties imposed by the nonholonomy of the integrator (1). Then, we regard the overall system as a cascaded one.
The controller equations are presented next in detail and farther below we explain the stabilization mechanism. Let

$$
\begin{aligned}
& u_{v i}=-k_{v i} \varphi_{i}\left(\theta_{i}\right)^{\top}\left(\bar{z}_{i}-\vartheta_{v i}\right), \\
& \ddot{\vartheta}_{v i}=-d_{v i} \dot{\vartheta}_{v i}-k_{v i}\left(\vartheta_{v i}-\bar{z}_{i}\right)-p_{v i} e_{v i} \\
& u_{\omega i}=-k_{\omega i}\left(\theta_{i}-\vartheta_{\omega i}\right)+\alpha_{i}\left(t, \theta_{i}, e_{v i}\right), \\
& \ddot{\vartheta}_{\omega i}=-d_{\omega i} \dot{\vartheta}_{\omega i}-k_{\omega i}\left(\vartheta_{\omega i}-\theta_{\omega i}\right)-p_{\omega i} e_{\omega i},
\end{aligned}
$$

where

$$
\begin{aligned}
e_{v i} & :=\sum_{j \in \mathcal{N}_{i}} a_{i j}\left[\vartheta_{v i}-\vartheta_{v j}\right] \\
e_{\omega i} & :=\sum_{j \in \mathcal{N}_{i}} a_{i j}\left[\vartheta_{\omega i}-\vartheta_{\omega j}\right],
\end{aligned}
$$

the constants $k_{v i}$ and $k_{\omega i}>0$ are plant-controller interconnection gains; $p_{v i}$ and $p_{\omega i}>0$ are proportional gains; $d_{v i}$ and $d_{\omega i}>0$ are damping gains and the term $\alpha_{i}$ consists in a $\delta$ persistently exciting function [8] that is defined and explained farther below.

The resulting closed-loop equations, have the forms previously discussed and, moreover, form a cascaded system.

$$
\begin{aligned}
\sum_{v_{i}}:\left\{\begin{aligned}
\dot{\bar{z}}_{i} & =\varphi_{i}\left(\theta_{i}\right) v_{i} \\
\dot{v}_{i} & =-k_{v i} \varphi_{i}\left(\theta_{i}\right)^{\top}\left(\bar{z}_{i}-\vartheta_{v i}\right), \\
\ddot{\vartheta}_{v i} & =-d_{v i} \dot{\vartheta}_{v i}-k_{v i}\left(\vartheta_{v i}-\bar{z}_{i}\right)-p_{v i} e_{v i}
\end{aligned}\right. \\
\sum_{\omega_{i}}:\left\{\begin{aligned}
\dot{\theta}_{i} & =\omega_{i} \\
\dot{\omega}_{i} & =-k_{\omega i}\left(\theta_{i}-\vartheta_{\omega i}\right)+\alpha_{i}\left(t, \theta_{i}, e_{v i}\right), \\
\ddot{\vartheta}_{\omega i} & =-d_{\omega i} \dot{\vartheta}_{\omega i}-k_{\omega i}\left(\vartheta_{\omega i}-\theta_{i}\right)-p_{\omega i} e_{\omega i} .
\end{aligned}\right.
\end{aligned}
$$

Note that each of the systems $\Sigma_{v i}$ and $\Sigma_{\omega i}$ is reminiscent of the closed-loop system equations (9)-(10) previously described, albeit certain differences. The most obvious is the function $\varphi_{i}$ in (15a) which stems from the nonholonomic constraints. Therefore, as opposed to the simple expression (10) which may be regarded as a spring force hinging the variables $q_{i}$ and $\vartheta_{i}$, the control law $u_{v i} \in \mathbb{R}^{2}$ in (12a) must also account for the robot kinematics, whence Eq. (15b).

Now, since the system (1a)-(2a) is not stabilizable to a set-point via smooth time-invariant feedback, our controller relies on the external function of time $f(t)$, which excites the system's modes $-c f$. [8]. More precisely, the function $\alpha_{i}$ is a $\delta$-persistently exciting function defined as

$$
\alpha_{i}\left(t, \theta_{i}, e_{v i}\right):=k_{\alpha i} f_{i}(t) \varphi_{i}\left(\theta_{i}\right)^{\perp \top}\left(\vartheta_{v i}-\bar{z}_{i}\right),
$$

where $k_{\alpha i}>0, \varphi_{i}\left(\theta_{i}\right)^{\perp}=\left[\begin{array}{ll}-\sin \left(\theta_{i}\right) & \cos \left(\theta_{i}\right)\end{array}\right]^{\top}$ is the annihilator of $\varphi_{i}$, i.e., $\varphi_{i}\left(\theta_{i}\right)^{\top} \varphi_{i}\left(\theta_{i}\right)^{\perp}=\varphi_{i}\left(\theta_{i}\right)^{\perp \top} \varphi_{i}\left(\theta_{i}\right)=0$ and $f_{i} \in \mathcal{C}^{2}\left(\mathbb{R}_{\geq 0}, \mathbb{R}\right)$ is designed such that $f_{i}, \dot{f}_{i}, \ddot{f}_{i} \in \mathcal{L}_{\infty}$, $\lim _{t \rightarrow \infty} f_{i}(t) \neq 0$, and $\lim _{t \rightarrow \infty} \dot{f}_{i}(t) \neq 0$. For instance a simple multi-periodic function may be employed, but not necessarily.

Remark 1 The function $\alpha_{i}$ plays a fundamental role in the controller to overcome the difficulties imposed by the nonholonomic constraints. For the purpose of argument, let us consider $f_{i}(t)$ as a multi-periodic function and let us replace $\theta_{i}$ with $\theta_{i}(t)$, that is, the state trajectories. The control mechanism resides on the fact that if (and only if) $\varphi\left(\theta_{i}(t)\right)$ excites all the modes in $\Sigma_{v i}$, one may establish the convergence of $e_{v i} \rightarrow 0$ 
and, hence, of $\alpha_{i}\left(t, \theta_{i}, e_{v i}(t)\right)$. In other words, consensus is not reached for the systems $\Sigma_{\omega i}$ as long as the consensus errors $e_{v i}$ persist away from zero, while the same oscillations induced into $\theta_{i}(t)$ ensure the convergence of $e_{v i}$. This stabilization mechanism is that of the so-called $\delta$-persistently exciting controllers [8], [23].

On the other hand, $\Sigma_{\omega i}$ consists in a linear time-invariant (stable) system as (9)-(10), but it is interconnected to $\Sigma_{v i}$ through the term $\alpha_{i}\left(t, \theta_{i}, e_{v i}\right)$. In turn, $\alpha_{i}$ is uniformly bounded in $t$ and $\theta_{i}$ and it is linear in $\left(\vartheta_{v i}-\bar{z}_{i}\right)$. This is important for the purpose of analyzing the systems $\Sigma_{v i}$ and $\Sigma_{\omega i}$ as a cascade. From such perspective, $\Sigma_{\omega i}$ corresponds to a system like (9)(10) being perturbed by the "input" $\alpha_{i}$.

Remark 2 Strictly speaking, the dependence of $\Sigma_{v i}$ on $\varphi_{i}\left(\theta_{i}\right)$, in which $\theta_{i}$ is a state variable of $\Sigma_{\omega i}$ establishes a feedback interconnection, but accounting for the fact $\varphi\left(\theta_{i}\right)$ is bounded, one may consider the overall system as a cascade of nonlinear time-varying systems [22].

The previous rationale leads to the following fact, which constitutes our main result.

Proposition 1 Consider a group of $N$ vehicles, each modeled by the equations (1)-(2) and in closed loop with the controller (12)-(17). Assume that they are interconnected over a network with an undirected, static and connected graph. Assume, further, that the control gains $k_{v i}, k_{\omega i}, d_{v i}, d_{\omega i}, p_{v i}$, and $p_{\omega i}$ are all positive and the function $\dot{f}_{i}(t)$ is persistently exciting, i.e., there exist $T$ and $\mu>0$ such that

$$
\int_{t}^{t+T} \dot{f}_{i}(s)^{2} d s \geq \mu \quad \forall t \geq 0 .
$$

Then, the formation-consensus control goal is achieved, that is, the expressions in (4) and (5) hold.

\section{ProOF OF Proposition 1}

The proof relies on Lyapunov theory and Barbălat's Lemma which (we recall for convenience that) states that if a function $t \mapsto g(t)$ is uniformly continuous (e.g., if $\dot{g}$ is bounded) and the limit of its integral exists and is finite, then $g(t) \rightarrow 0$ asymptotically. We also use the fact (which follows from Barbălat's Lemma) that if a function $g(t)$ is bounded $\left(g \in \mathcal{L}_{\infty}\right)$, squareintegrable $\left(g \in \mathcal{L}_{2}\right)$ and admits a bounded derivative $\left(\dot{g} \in \mathcal{L}_{\infty}\right)$ then $g(t) \rightarrow 0$ as $t \rightarrow \infty$

First, consider the "energy" function for the forwardvelocity dynamics, $\Sigma_{v i}$,

$$
\mathcal{E}_{v i}:=\frac{1}{2}\left[v_{i}^{2}+\left|\dot{\vartheta}_{v i}\right|^{2}+k_{v i}\left|\vartheta_{v i}-\bar{z}_{i}\right|^{2}\right]
$$

which is positive definite and radially unbounded in $v_{i}$, $\dot{\vartheta}_{v i}$, and $\left(\vartheta_{v i}-\bar{z}_{i}\right)$. The total derivative of this function along the trajectories of (15) yields $\dot{\mathcal{E}}_{v i}=-d_{v i}\left|\dot{\vartheta}_{v i}\right|^{2}-$ $p_{v i} \sum_{j \in \mathcal{N}_{i}} a_{i j} \dot{\vartheta}_{v i}^{\top}\left(\vartheta_{v i}-\vartheta_{v j}\right)$.

Then, for the complete swarm of networked vehicles, consider the Lyapunov function candidate

$$
\mathcal{V}:=\sum_{i \leq N}\left[\frac{1}{p_{v i}} \mathcal{E}_{v i}+\frac{1}{4} \sum_{j \in \mathcal{N}_{i}} a_{i j}\left|\vartheta_{v i}-\vartheta_{v j}\right|^{2}\right] .
$$

Note that there exist $\sigma_{1}, \sigma_{2}>0$ such that, defining $\xi_{v}:=$ $\left[\cdots v_{i} \cdots \dot{\vartheta}_{v i} \cdots\left(\vartheta_{v i}-\bar{z}_{i}\right) \cdots\left(\vartheta_{v i}-\vartheta_{v j}\right) \cdots\right]^{\top}$, we have

$$
\sigma_{1}\left|\xi_{v}\right|^{2} \leq \mathcal{V}\left(\xi_{v}\right) \leq \sigma_{2}\left|\xi_{v}\right|^{2}
$$

Moreover, using [24, Lemma 6.1] one finds that the total derivative of $\mathcal{V}$ yields

$$
\dot{\mathcal{V}}=\sum_{i \leq N}\left[\frac{1}{p_{v i}} \dot{\mathcal{E}}_{v i}+\sum_{j \in \mathcal{N}_{i}} a_{i j} \dot{\vartheta}_{v i}^{\top}\left(\vartheta_{v i}-\vartheta_{v j}\right)\right]
$$

hence, $\dot{\mathcal{V}} \leq-\sum_{i \leq N} \lambda_{v i}\left|\dot{\vartheta}_{v i}\right|^{2} \leq 0$ with $\lambda_{v i}:=d_{v i} / p_{v i}$. In view of (20), integrating on both sides of $\dot{\mathcal{V}} \leq 0$ it follows that $\xi_{v} \in$ $\mathcal{L}_{\infty}$, while integrating on both sides of $\dot{\mathcal{V}} \leq-\sum_{i<N} \lambda_{v i}\left|\dot{\vartheta}_{v i}\right|^{2}$ and using the boundedness of $\xi_{v}$ just established, it follows that $\dot{\vartheta}_{v i} \in \mathcal{L}_{2}$ for all $i \leq N$. This and the fact that $\dot{\vartheta}_{v i}$, $\ddot{\vartheta}_{v i} \in \mathcal{L}_{\infty}$ imply that $\dot{\vartheta}_{v i} \rightarrow 0$.

Furthermore, the convergence of $\ddot{\vartheta}_{v i}$ and $\vartheta_{v i}^{(3)}$ also follows from applying successively Barbălat's Lemma. For this we differentiate twice on both sides of (15c) to obtain

$$
\begin{aligned}
\vartheta_{v i}^{(3)} & =-d_{v i} \ddot{\vartheta}_{v i}-k_{v i}\left[\dot{\vartheta}_{v i}-\dot{\bar{z}}_{i}\right]-p_{v i} \dot{e}_{v i} \\
\vartheta_{v i}^{(4)} & =-d_{v i} \vartheta_{v i}^{(3)}-k_{v i}\left[\ddot{\vartheta}_{v i}-\ddot{\bar{z}}_{i}\right]-p_{v i} \ddot{e}_{v i} .
\end{aligned}
$$

From the latter equations, Eqs. (15), and the boundedness of $\xi_{v}$ it follows that all the terms in (21) and (22) are bounded (this involves the boundedness of $\omega_{i}$ which is established farther below). Therefore, $\ddot{\vartheta}_{v i}$ and $\vartheta_{v i}^{(3)}$ are uniformly continuous. Moreover,

$$
\lim _{t \rightarrow \infty} \int_{0}^{t} \ddot{\vartheta}_{v i}(\sigma) d \sigma=\lim _{t \rightarrow \infty} \dot{\vartheta}_{v i}(t)-\dot{\vartheta}_{v i}(0)=-\dot{\vartheta}_{v i}(0)
$$

so, by Barbălat's Lemma it follows that $\ddot{\vartheta}_{v i} \rightarrow 0$. A similar argument applies to establish that $\vartheta_{v i}^{(3)} \rightarrow 0$. Using once again (21) we conclude that $\dot{\bar{z}}_{i}$ and, hence, $v_{i}$ also converges to zero asymptotically.

Let us consider next the orientation dynamics (16), albeit without interconnection. That is, for the time being let $\alpha_{i} \equiv 0$ for all $i \leq N$. Akin to case of $\Sigma_{v i}$, we introduce the "energy" function $\mathcal{E}_{\omega i}:=\frac{1}{2}\left[\omega_{i}^{2}+\dot{\vartheta}_{\omega i}^{2}+k_{\omega i}\left(\vartheta_{\omega i}-\theta_{i}\right)^{2}\right]$. Its total derivative along the trajectories of (16) yields

$$
\dot{\mathcal{E}}_{\omega i}=-d_{\omega i}\left|\dot{\vartheta}_{\omega i}\right|^{2}-p_{\omega i} \sum_{j \in \mathcal{N}_{i}} a_{i j} \dot{\vartheta}_{\omega i}^{\top}\left(\vartheta_{\omega i}-\vartheta_{\omega j}\right) .
$$

Hence, in turn, the total derivative of the Lyapunov-function candidate

$$
\mathcal{W}:=\sum_{i \leq N}\left[\frac{1}{p_{\omega i}} \mathcal{E}_{\omega i}+\frac{1}{4} \sum_{j \in \mathcal{N}_{i}} a_{i j}\left(\vartheta_{\omega i}-\vartheta_{\omega j}\right)^{2}\right]
$$

satisfies $\dot{\mathcal{W}} \leq-\sum_{i \leq N} \lambda_{\omega i} \dot{\vartheta}_{\omega_{i}}^{2} \leq 0$, with $\lambda_{\omega i}:=d_{\omega i} / p_{\omega i}$. Proceeding as with $\mathcal{V}$ above, we conclude that $\xi_{\omega}:=$ $\left[\cdots \omega_{i} \cdots \dot{\vartheta}_{\omega i} \cdots\left(\vartheta_{\omega i}-\theta_{i}\right) \cdots\left(\vartheta_{\omega i}-\vartheta_{\omega j}\right) \cdots\right]^{\top}$ is bounded and $\dot{\vartheta}_{\omega i} \in \mathcal{L}_{2}$. Therefore, on one hand, the consensus errors $e_{\omega i}$ are also bounded and, on the other, $\dot{\vartheta}_{\omega i} \rightarrow 0$. From this 
and the successive application of Barbălat's Lemma it is also concluded that $\dot{\vartheta}_{\omega i}, \ddot{\vartheta}_{\omega i}$, and $\vartheta_{\omega i}^{(3)} \rightarrow 0$. In turn, we see from

$$
\vartheta_{\omega i}^{(3)}=-d_{\omega i} \ddot{\vartheta}_{\omega i}-k_{\omega i}\left(\dot{\vartheta}_{\omega i}-\omega_{i}\right)-p_{\omega i} \dot{e}_{\omega i}
$$

that $\omega_{i} \rightarrow 0$, as required in (5). Therefore, since

$$
\lim _{t \rightarrow \infty} \int_{0}^{t} \dot{\omega}_{i}(\sigma) d \sigma=\lim _{t \rightarrow \infty} \omega_{i}(t)-\omega_{i}(0)=-\omega_{i}(0),
$$

and $\dot{\omega}_{i}$ is uniformly continuous (because $\ddot{\omega}_{i}=-k_{\omega i}\left(\omega_{i}-\dot{\vartheta}_{\omega i}\right.$ is bounded) it follows from Barbălat's Lemma that $\dot{\omega}_{i}$ also converges to zero and, therefore, from (16b) (recall that $\alpha_{i} \equiv$ 0 ), we obtain that $\left(\theta_{i}-\vartheta_{\omega i}\right) \rightarrow 0$. Next, from (16c), it follows that $e_{\omega i} \rightarrow 0$. Equivalently, the second limit in (5) holds.

All the previous arguments hold provided that $\alpha_{i} \equiv 0$. If this is not the case, we remark that since, $f_{i}, v_{v i}-\bar{z}_{i}$ and $\varphi_{i}^{\perp}$ are bounded along all trajectories, so is $\alpha_{i}$ - see Eq. (17). This and the fact that $\Sigma_{\omega i}$ is a marginally stable linear time-varying system suggest, by Proposition 3 in [25], that $\dot{\omega}_{i}, \omega_{i}, \dot{\vartheta}_{\omega i}, \ddot{\vartheta}_{\omega i} \in \mathcal{L}_{\infty}$ and so $\theta_{i}-\vartheta_{\omega i}$ and $e_{\omega i}$ are also bounded. Furthermore, invoking once more Proposition 3 in [25] it follows that the previous conclusions hold provided that $\alpha_{i} \rightarrow 0$, which holds if and only if $\left(\vartheta_{v i}-\bar{z}_{i}\right) \rightarrow 0$.

To show that $\left(\vartheta_{v i}-\bar{z}_{i}\right) \rightarrow 0$, we first observe that the fact that $v_{i} \rightarrow 0$ and the boundedness of $\xi_{v}(t)$ implies that, also $\dot{v}_{i} \rightarrow 0$ and $\ddot{v}_{i} \rightarrow 0$. This follows from Barbălat's Lemma, noting that

$$
\lim _{t \rightarrow \infty} \int_{0}^{t} \dot{v}_{i}(\sigma) d \sigma=\lim _{t \rightarrow \infty} v_{i}(t)-v_{i}(0)=-v_{i}(0)
$$

all the terms on the right hand side of

$$
\ddot{v}_{i}=-k_{v i} \varphi_{i}\left(\theta_{i}\right)^{\top}\left[\dot{\bar{z}}_{i}-\dot{\vartheta}_{v i}\right]-k_{v i} \omega_{i} \varphi_{i}\left(\theta_{i}\right)^{\perp \top}\left(\bar{z}_{i}-\vartheta_{v i}\right)
$$

are bounded. Then $\ddot{v}_{i} \in \mathcal{L}_{\infty}$ and $\dot{v}_{i} \rightarrow 0$. A similar reasoning leads to concluding that $\ddot{v}_{i} \rightarrow 0$. We also recall that $\omega_{i} \rightarrow 0$, hence

$$
\lim _{t \rightarrow \infty} \int_{0}^{t} \dot{\omega}(s) d s=-\omega_{i}(0)<\infty
$$

and since $\ddot{\omega}_{i} \in \mathcal{L}_{\infty}$ we obtain, from Barbălat's Lemma that $\dot{\omega}_{i} \rightarrow 0$. From a similar argument we conclude that $\ddot{\omega}_{i} \rightarrow 0$. Now, differentiating on both sides of (16b) we obtain

$$
\begin{aligned}
\ddot{\omega}_{i}= & -k_{\omega i}\left(\omega_{i}-\dot{\vartheta}_{\omega i}\right)+k_{\alpha i} \dot{f}_{i}(t) \varphi_{i}\left(\theta_{i}\right)^{\perp \top}\left(\vartheta_{v i}-\bar{z}_{i}\right) \\
& -k_{\alpha i} \omega_{i} f_{i}(t) \varphi_{i}\left(\theta_{i}\right)^{\top}\left(\vartheta_{v i}-\bar{z}_{i}\right)+k_{\alpha i} f_{i}(t) \varphi_{i}\left(\theta_{i}\right)^{\perp \top} \dot{\vartheta}_{v i} .
\end{aligned}
$$

From this equation and (15) we obtain that

$$
\lim _{t \rightarrow \infty} \varphi_{i}\left(\theta_{i}(t)\right)^{\top}\left(\bar{z}_{i}(t)-\vartheta_{v i}(t)\right)=0
$$

and

$$
\lim _{t \rightarrow \infty} \dot{f}_{i}(t) \varphi_{i}\left(\theta_{i}(t)\right)^{\perp \top}\left(\bar{z}_{i}(t)-\vartheta_{v i}(t)\right)=0,
$$

which, together, imply that $\left(\bar{z}_{i}-\vartheta_{v i}\right) \rightarrow 0$ since $\dot{f}_{i}$ is persistently exciting by assumption - see Ineq. (18).

Next, from (15c) and the fact that $\ddot{\vartheta}_{v i} \rightarrow 0, \dot{\vartheta}_{v i} \rightarrow 0$, it also follows that if $\left(\vartheta_{v i}-\bar{z}_{i}\right) \rightarrow 0$ then $e_{v i} \rightarrow 0$ for all $i \leq N$. This implies that $\vartheta_{v i} \rightarrow \vartheta_{v j}$ or, equivalently, that $\vartheta_{v i} \rightarrow z_{c} \equiv$ const. for all $i \leq N$. Thus, the second limit in (4) also holds. This completes the proof.

\section{Simulation RESUltS}

In lack of an experimental set-up, we have performed extensive numerical simulations to test the performance of our controller. The simulations were performed using Simulink ${ }^{\circledR}$ of Matlab ${ }^{\circledR}$. In the simulation scenario we have employed six drive-wheel robots of the type TurtleBot 3 Waffle Pi (see http://www.robotis.us/turtlebot-3-waffle-pi/) exchanging information through a wireless network. The specifications of these robots is as follows $-c f$. Eqs. (1)-(2): $m=1.9, I=0.5$, $R=0.15$, and $r=0.033$.

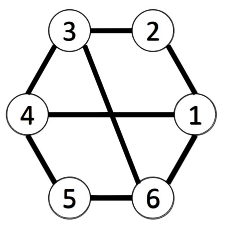

\begin{tabular}{c|cccccc} 
Rовот & 1 & 2 & 3 & 4 & 5 & 6 \\
\hline$\delta_{x i}$ & 2 & 1 & -1 & -2 & -1 & 1 \\
$\delta_{y i}$ & 0 & 2 & 2 & 0 & -2 & -2 \\
\hline
\end{tabular}

Fig. 3. Communication topology and hexagonal desired formation pattern (left). The latter is defined as an hexagon, determined by the distances $\delta_{x i}$ and $\delta_{y i}$ as per in the table on the right.

It is supposed that the robots communicate according to the graph showed in Fig. 3. The graph is intended to illustrate the communication topology only, but it also serves in illustrating the formation pattern that the robots are required to converge to in one of the simulated scenarii, described farther below. Other formation patterns (straight line, rectangle) can be easily incorporated by changing $\delta_{x i}$ and $\delta_{y i}$. In all cases, neither the center of the formation nor the final orientation are predefined. Recall that the consensus formation problem solved here may be considered as an "initialization" step of a more complex advance-in-formation mission, in which the robots are firstly "summoned up" to a rendezvous point.

In the simulation scenario described below the initial positions and orientations for the robots are given by

$$
\begin{aligned}
& x_{i}(0):=\left[\begin{array}{llllll}
8 & 2 & 2 & -2 & 1 & 4
\end{array}\right]^{\top}, \quad y_{i}(0):=\left[\begin{array}{llllll}
7 & 13 & 9 & 6 & 3 & 4
\end{array}\right]^{\top}, \\
& \theta(0)=\left[\frac{\pi}{2} 0-\frac{\pi}{8} \frac{\pi}{8}-\frac{\pi}{8}-\frac{\pi}{8}\right]^{\top} .
\end{aligned}
$$

The initial positions may be well appreciated in Fig. 4, marked with coloured ' $\circ$ '. For simplicity, since all robots are assumed to be identical, we have used the same control gains for all the controllers. Hence, for each robot, we fix the latter as follows: we choose relatively high "spring stiffness" $k_{v}=100$ and $k_{w}=100$. Then, the control gains are set to $p_{v i}=p_{v}$, $p_{\omega i}=p_{\omega}, d_{v i}=d_{v}$, and $d_{\omega i}=d_{\omega}$ for all $i \leq 6$ in a way that the corresponding second-order systems $\ddot{z}+d_{v} \dot{z}+p_{v} z=0$ and $\ddot{z}+d_{\omega} \dot{z}+p_{\omega} z=0$. We used $p_{v}=5, p_{w}=5, d_{v}=15$, and $d_{w}=15$. The functions $f_{i}(t)$ are also defined equally for all robots and persistently exciting. For their definition, we borrow inspiration from the literature on adaptive control and systems' identification. It is well-known that in order to estimate the lumped constant parameters of a system one needs to use as many frequencies as there are unknown parameters. There is no theoretical foundation for the employment of such rule in the present context, but experience shows that a function $f$ having as many frequencies as robots in the network may 
produce satisfactory results. We used

$$
f(t)=3+\sin (t)+\cos (3 t)-\sin (4 t)-\cos (5 t)+\sin (0.5 t) \text {. }
$$

As it may be appreciated from Fig. 4, the robots converge to the desired formation pattern with a barycenter at $z_{c}=$ $[4.2,9.42]^{\top}$.

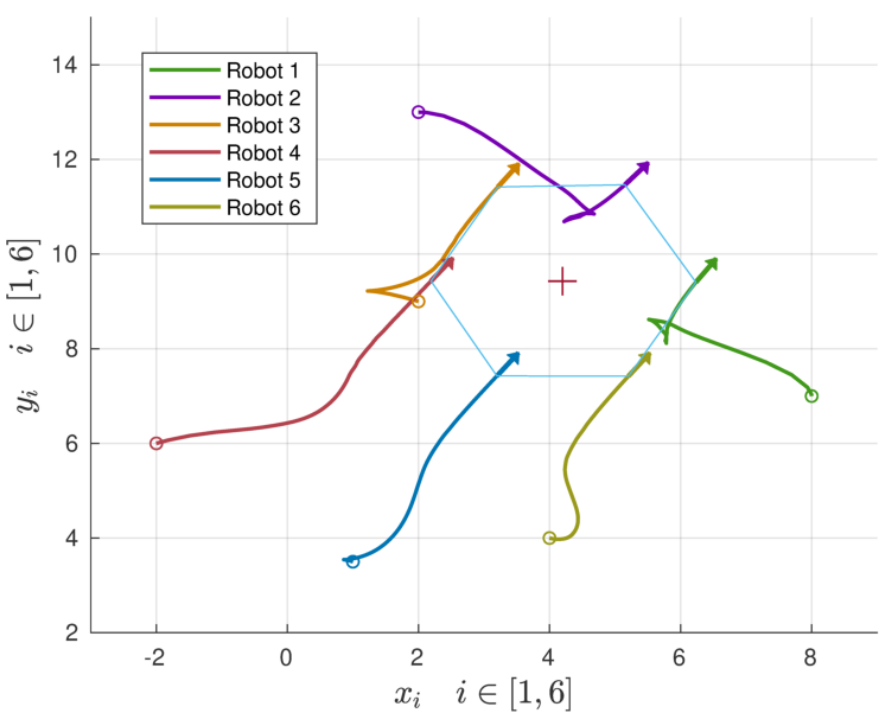

Fig. 4. Position trajectories in the Cartesian $\boldsymbol{x} \boldsymbol{y}$-plane.

The paths that the robots take is as expected. For instance, Robot 1 and Robot 2 communicate with each other and, therefore, tend to approach, as the other robots join the group, Robots 1 and 2 change to a consensual direction. The center of the formation pattern continuously changes as the robots move. The final orientations of all robots, reaching a consensual angle of $\theta_{c} \approx 1 \mathrm{rad}$, is also appreciated (represented by pointing arrows) in Fig. 4.

\section{CONCLUSIONS}

We have presented a simple and intuitive control approach to solve the difficult problem of leaderless consensus control of autonomous vehicles without velocity measurements. Our main controller relies on a simple dynamic controller with a clear physical meaning. Further research is focused on the incorporation of meaningful conditions stemming from the setup, such as the presence of time-varying delays, which are fairly common in wifi networks. In addition, the experimental testing of our findings, which has not been possible to carry out for lack of bench-marking equipment, is primordial.

\section{REFERENCES}

[1] X. Wang and Y. Hong, "Distributed observers for tracking a moving target by cooperative multiple agents with time delays," in 2009 ICCAS SICE, pp. 982-987, 2009.

[2] X. Liang, H. Wang, Y. Liu, W. Chen, and T. Liu, "Formation control of nonholonomic mobile robots without position and velocity measurements," IEEE Trans. on Robotics, vol. 34, no. 2, pp. 434-446, 2018.
[3] W. Mao, C. Wang, W. Chen, and X. Li, "Observer-based consensus design for multi-agent systems with unavailable velocities of leader and followers," in Proceedings of the 32nd Chinese Control Conference, pp. 7030-7033, 2013.

[4] W. Dong, "Distributed observer-based cooperative control of multiple nonholonomic mobile agents," International Journal of Systems Science, vol. 43, no. 5, pp. 797-808, 2012.

[5] G. Chen, Y. Yue, and Y. Song, "Finite-time cooperative-tracking control for networked Euler-Lagrange systems," IET Control Theory and Applications, vol. 7, no. 11, pp. 1487-1497, 2013.

[6] E. Montijano, J. Thunberg, X. Hu, and C. Sagüés, "Epipolar visual servoing for multirobot distributed consensus," IEEE Trans. on Robotics, vol. 29, no. 5, pp. 1212-1225, 2013.

[7] Y. Cheng, R. Jia, H. Du, G. Wen, and W. Zhu, "Robust finite-time consensus formation control for multiple nonholonomic wheeled mobile robots via output feedback," International Journal of Robust and Nonlinear Control, vol. 28, no. 6, pp. 2082-2096, 2018

[8] E. Nuño, A. Loría, A. T. Hernández, M. Maghenem, and E. Panteley, "Distributed consensus-formation of force-controlled nonholonomic robots with time-varying delays," Automatica, no. 120, p. 109114, 2020.

[9] D. Dimarogonas and K. Kyriakopoulos, "On the rendezvous problem for multiple nonholonomic agents," IEEE Transactions Automatic Control, vol. 52, pp. 916-922, May 2007.

[10] M. Maghenem, Stability and stabilization of networked time-varying systems. PhD thesis, Univ Paris Saclay, Gif sur Yvette, 2017. https://tel.archives-ouvertes.fr/tel-01596158.

[11] Z. Lin, B. Francis, and M. Maggiore, "Necessary and sufficient graphical conditions for formation control of unicycles," IEEE Trans. on Automatic Control, vol. 50, no. 1, pp. 121-127, 2005.

[12] W. Ren and Y.-Q. Chen, "Leaderless formation control for multiple autonomous vehicles," in AIAA Guidance, Navigation, and Control Conference and Exhibit, pp. 2006-6069, 2006.

[13] S. Zhao, "Affine formation maneuver control of multiagent systems," IEEE Transactions on Automatic Control, vol. 63, no. 12, pp. 4140$4155,2018$.

[14] J. Xi, Z. Shi, and Y. Zhong, "Output consensus analysis and design for high-order linear swarm systems: Partial stability method," Automatica, vol. 48, pp. 2335-2343, 2012.

[15] A. Abdessameud and A. Tayebi, "On consensus algorithms for doubleintegrator dynamics without velocity measurements and with input constraints," Systems \& Control Letters, vol. 59, no. 12, pp. 812 - 821, 2010.

[16] H. A. Poonawala, A. C. Satici, and M. W. Spong, "Leader-follower formation control of nonholonomic wheeled mobile robots using only position measurements," in 2013 9th Asian Control Conference (ASCC), pp. 1-6, June 2013.

[17] A. T. Hernández, A. Loría, E. Nuño, and E. Panteley, "Consensusformation control of nonholonomic robots without velocity measurements," in Proc. European Control Conference (ECC), (St. Petersburg, Russia), pp. 674-679, 2020.

[18] S. G. Tzafestas, Introduction to mobile robot control. Elsevier Inc, First ed., 2013.

[19] W. Ren and R. W. Beard, Distributed consensus in multivehicle cooperative control. Springer verlag, 2005.

[20] R. Kelly, "A simple set-point robot controller by using only position measurements," in Proc. 12th. IFAC World Congress, vol. 6, (Sydney, Australia), pp. 173-176, 1993.

[21] E. Nuño and R. Ortega, "Achieving consensus of Euler-Lagrange agents with interconnecting delays and without velocity measurements via passivity-based control," IEEE Trans. on Control Systems Technology, vol. 26, no. 1, pp. 222-232, 2018.

[22] A. Loría, "From feedback to cascade-interconnected systems: Breaking the loop," in Proc. 47th. IEEE Conf. Decision Contr., (Cancun, Mex.), pp. 4109-4114, 2008.

[23] A. Loría, E. Panteley, and K. Melhem, "UGAS of skew-symmetric timevarying systems: application to stabilization of chained form systems," European J. of Contr., vol. 8, no. 1, pp. 33-43, 2002.

[24] Y. Cao and W. Ren, Distributed Coordination of Multi-agent Networks: Emergent Problems, Models, and Issues. Springer-Verlag, 2011.

[25] H. Wang, "Differential-cascade framework for consensus of networked lagrangian systems," Automatica, vol. 112, p. 108620, 2020. 